\title{
Fabrication of Locally Made Bucket Centrifuge in Medical Laboratory Science Department - Ebonyi State University, Abakaliki
}

Udeajah, V.N.

Department of Medical laboratory Science, Ebonyi State University, Abakaliki, Nigeria.

Email: vakadujah45@gmail.com

DOI: http://doi.org/10.38177/ajast.2021.5311

Copyright: () 2021 Udeajah, V.N. This is an open access article distributed under the terms of the Creative Commons Attribution License, which permits unrestricted use, distribution, and reproduction in any medium, provided the original author and source are credited.

The bucket centrifuge is an essential equipment used in the laboratory for spinning of samples such as blood and urine. Imported bucket centrifuge can be expensive, the need for improvisation or fabricate locally made ones using available resources that are less expensive became necessary. This bucket centrifuge was locally fabricated in the department of Medical laboratory science in Ebonyi State University- Abakaliki to enable the students have an understanding of Biomedical engineering and bridge the gap between theory and practicals involved in their professional practice.

Keywords: Bucket centrifuge, Spinning of samples, Improvisation and Biomedical engineering studies.

\section{Introduction to Centrifuge}

The centrifuge is equipment used in the laboratory for spinning of samples. The fluid substance sediment according to their density or size, after centrifuging the supernatant is usually discarded and the sediment is used to carry out diagnosis. The centrifuge are of various sizes and shapes, with different speed or revolution per minute. Various materials like aluminum, metal, or stainless can be used to fabricate the centrifuge. It has a mechanical and an electrical part respectively.

\section{Principle of Bucket Centrifuge}

"The centrifuge works using the sedimentation principle, where the centrifugal acceleration causes denser substances and particles to move outward in the radial direction. At the same time, objects that are less dense are displaced and move to the center".

\section{Principle of Operation}

$\square$ A centrifuge is a piece of equipment, generally driven by an electric motor, that puts an object in rotation around a fixed axis, applying a force perpendicular to the axis to separate substances of different densities.

$\square$ Tubes in the centrifuge are tilted so centrifugal force can pull denser substances towards the bottom of the tube.

$\square$ Relative Centrifugal Force (RCF) measures acceleration applied to the sample

- $R C F=\left(r^{*} \omega^{2}\right) / g$
a $r$ distance from center or motor to sample
$\square=$ angular velocity (radians/ second)

$\omega=$ angular
$\mathrm{g}=9.8 \mathrm{~m} / \mathrm{s}^{2}$

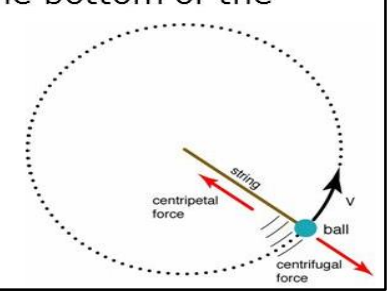

Courtesy: microbesnotes.com

Types of Bucket Centrifuge: There are multiple types of centrifuge, which can be classified by intended use or by rotor design. However, there are four major types of centrifuges: 
1. Small Bench Centrifuges: They are used to collect small amount of material that sediment like yeast cells, erythrocytes and so on. They have maximum relative centrifugal field of 3000-7000g.

2. Large Capacity Refrigerated Centrifuges: They have refrigerated rotor chamber for varying size. They can go up to maximum of $6500 \mathrm{~g}$ and use to sediment or collect the substances that sediment rapidly like erythrocytes, yeast cells and so on.

3. High Speed Refrigerated Centrifuges: They can generate speed of about $60000 \mathrm{~g}$ and are used to collect micro-organism, cellular debris, large cellular organelles and proteins.

4. Ultra Centrifuges: They are of two kinds, namely

(a) Preparative ultracentrifuge and

(b) Analytical ultracentrifuge.

Both can produce a relative centrifugal force of about $50000 \mathrm{~g}-60000 \mathrm{~g}$.

\section{Types by rotor design}

(a) Fixed-angle centrifuges, which are designed to hold the sample containers at constant angle relative to the central axis.

(b) Swinging head (or swinging bucket) centrifuges, which has a hinge where sample containers are attached to the central rotor. This allows all of the samples to swing outwards as the centrifuge is spun.

(c) Continuous tubular centrifuges, which do not have individual sample vessels and are used for high volume applications.

\section{Application of Bucket Centrifuge}

The bucket centrifuge comes in various design and shape, and can be manually operated or electrically operated. It has a regulator that is used to control the speed - for electrically operated bucket centrifuge. Test tubes or sample container are carefully placed in the sample holder and balanced to aid easy spinning and to achieve good results. In a laboratory centrifuge that uses sample tubes, the radial acceleration causes denser particles to settle to the bottom of the tube, while low-density substances rise to the top. It has an electric motor that spins the sample. It is used in almost all units of medical laboratory science.

\section{Standard Operating Procedure of Bucket Centrifuge}

Aim: To lay down the procedure for operation of bucket centrifuge.

\section{Procedure:}

(1) Connect the equipment to the power source.

(2) Open the equipment and arrange the test tube or sample containers in the test tube holder ensuring it balances with another.

(3) "On" the equipment by pressing the power button. 


\section{AJAST}

Asian Journal of Applied Science and Technology (AJAST)

Volume 5, Issue 3, Pages 120-125, July-September 2021

(4) Using the timer, set the number of minutes you want the equipment to spin.

(5) Use your left hand to press "start" on the timer, and your right hand to "on" the regulator at the same time.

(6) Once the time has elapsed, "off” the regulator and put "off” the equipment.

(7) Allow the equipment to stop spinning completely before removing the samples.

(8) After use, switch off and disconnect from the power source.

\section{Parts and Functions of the Bucket Centrifuge}

Metal Casing: This is used to fabricate the body of the centrifuge that is housing the electric motor in position and other electrical components like timer, regulator, etc. It can be made of stainless steel or aluminum to avoid rusting.

Electric Motor: This is the machine for converting electrical energy to mechanical energy. The electric motor rotates the shaft with a maximum speed required for the centrifuge.

Regulator: This is used to control the speed of the machine, either by increasing the speed or reducing it.

Timer: This is used to monitor the time for centrifuging.

Sample Holder: It is fixed on electric motor, it is where the test tubes containing samples are placed for spinning.

Power Button or Switch: This is used to generate power after the equipment is plugged to the power source.

Plug: This is where the wire is connected or joined before it is plugged to the power source.

Flex Wire [1.5 mm]: This is used for the entire connection of the various components of the centrifuge up to the point of its connection to the source of power, for the equipment to function.

Transformer: It is electrical device by which an alternating current of one voltage is changed to another voltage.

Diode: It is a semi-conductor that consists of a PN junction. It can also be a thermionic tube having two electrodes; used as a rectifier.

\section{Reasons for Fabrication of Bucket Centrifuge}

1. To reduce cost in terms of financial expenditure in buying ready-made materials.

2. It is a way of improvisation, which helps to bridge the gap between theoretical knowledge and practical.

3. To enable the lecturers and learners (students) to make proper use of their knowledge of biomedical engineering.

4. Active participation and sharing of ideas between the student and the lecturer can lead to the discovery of new innovations, which encourages higher exploits and much learning (Research).

\section{Materials and Methods}

\section{Materials}

1) Electric Motor

2) Regulator 

3) Power Button
4) Timer
5) Two yards of $1.5 \mathrm{~mm}$ flex wire
6) Metal Casing.
7) Sample Holder (Gas Burner)
8) Plug
9) Diode
10) Transformer (15 Volts)

\section{Methods}

a) A Market survey was made by selected individuals from the group to ascertain the price of the materials.

b) Financial contribution by group members was made after a specified amount was accepted by all the members.

c) The materials were purchased by the various members of the group.

d) The height and breadth of the metal sheet were measured: $1 \mathrm{ft}$. by $1.5 \mathrm{ft}$, and was coupled by a welder.

e) The electric motor was placed firmly on a metal material that is a bit elevated above the base.

f) The sample holder was placed carefully but firmly on the electric motor, to ensure it doesn't dangle while spinning. This ensures that the sample do not pour away during centrifuging.

g) Various openings were made for the various electrical components.

h) This was followed by electrical fixing and connections of the various components

i) The electric motor was connected to the transformer to step down and convert the A.C. voltage to D.C.

j) Diodes consisting of PN junction were used as rectifiers and were connected with the electric motor, transformer, regulator and switch (power button).

k) After that it is connected to the cable and joined to the plug.

1) The equipment was tested to ensure it was functional.

\section{Uses of Bucket Centrifuge}

The major use of the bucket centrifuge or centrifuge in general, is for the separation of substances according to their density. The centrifugal force applied is responsible for the degree of sedimentation.

\section{Samples that can be Spinned using Bucket Centrifuge}

\section{BLOOD 2. URINE}

\section{Advantages of fabricated bucket centrifuge over imported ones}

1) It is less expensive because of the use of local materials.

2) The fabricated bucket centrifuge is very easy to operate compared to imported ones. 


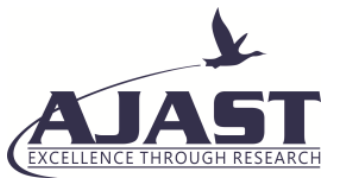

Asian Journal of Applied Science and Technology (AJAST)

Volume 5, Issue 3, Pages 120-125, July-September 2021

3) The fabrication encourages learners to higher exploits and can lead to the discovery of new knowledge for the lecturers and students.

\section{Disadvantages of fabricated bucket centrifuge over imported ones}

1) It can expose the teacher and the learner to some hazards.

2) It takes a lot of time both for teaching and learning of the

\section{Fabrication process}

1) Imported or ready-made bucket centrifuge, produces more accurate results than the locally fabricated ones.

2) Imported bucket centrifuge have more desired features than the locally fabricated ones.

\section{Care and Maintenance}

1. Centrifuge should be kept in a dry and airy environment.

2. Centrifuge should be kept in a vibration free bench.

3. Centrifuge should be kept away from direct sunlight and moist or humid environment.

4. Centrifuge should be serviced regularly according to manufacturers instruction, in order for it to last long.

5. Centrifuge should be carefully stored, covered and packed in a clean, cool, and airy environment if it won't be used for a long time.

6. The equipment should be allowed to stop spinning completely before removal of the sample.

\section{Snapshots}
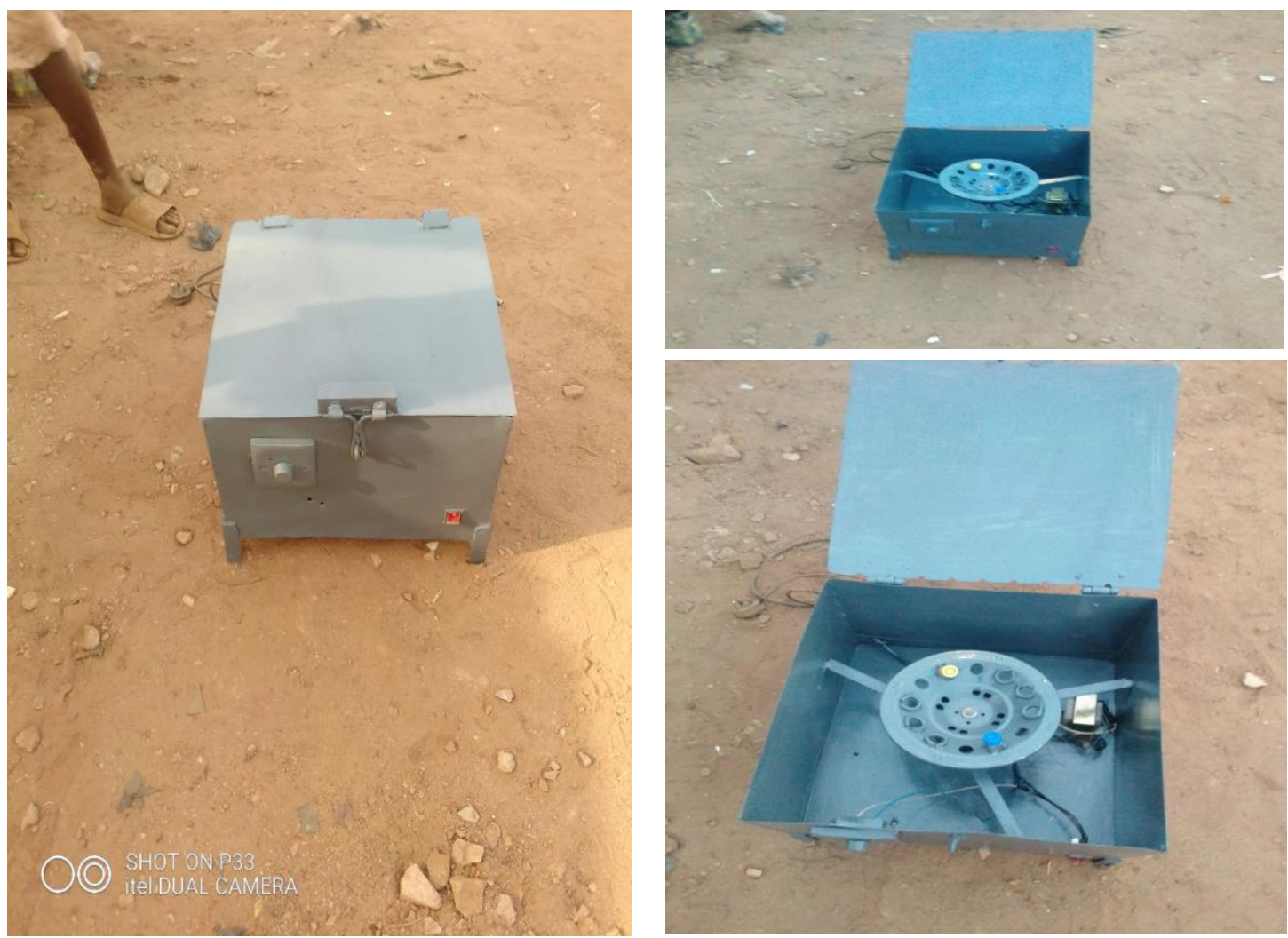


\title{
AJAST
}

Asian Journal of Applied Science and Technology (AJAST)

Volume 5, Issue 3, Pages 120-125, July-September 2021

\section{Conclusion \& Recommendation}

The fabrication of the bucket centrifuge requires careful planning, spirit of cooperation among members of the group, and careful study on how to put ideas and the materials together to produce functional equipment.

The project was a success, and there was maximum cooperation among group members. The ideas contributed by each member of the group lead to the production of a fair and functional equipment. Also all thanks to our kind and able lecturer, Engr. (Mrs.) Udeajah V.N., who ensured that the work was done at an affordable cost.

It is therefore recommended that construction of this type of Equipment, be encouraged in order to reduce cost of the instrument and make it more readily available in our laboratories.

\section{Acknowledgment}

The biomedical electronics engineering class are grateful to the staff of the ultramodern medical laboratory of the Department of Medical laboratory Science, EBSU, Abakaliki.

\section{Declarations}

\section{Source of Funding}

This research did not receive any grant from funding agencies in the public, commercial, or not-for-profit sectors.

\section{Competing Interests Statement}

The author declares no competing financial, professional and personal interests.

\section{Ethical Approval}

Based on Institutional guidelines.

\section{Consent for publication}

Author declares that she consented for the publication of this research work.

\author{
References \\ [1] www.microbes.com \\ [2] www.slidesnotes.com \\ [3] Udeajah, V.N. (2016). Biomedical engineering for B.MLS and PGD students, Agoms Printing Press, Afikpo, \\ Ebonyi State, Nigeria.
}

